\title{
CARACTERÍSTICAS CLÍNICO EPIDEMIOLÓGICAS DE LA INSUFICIENCIA CARDÍACA AGUDA EN UN HOSPITAL TERCIARIO DE LIMA, PERÚ
}

\author{
Marcos Pariona ${ }^{1,2, a}$, Pedro Antonio Segura Saldaña ${ }^{1, c}$, Marcos Padilla Reyes $^{1, c}$, Jorge Sebastián Reyes Villanes $^{1, b}$,
} Marcos Jáuregui Contreras ${ }^{1, \mathrm{~b}}$, Germán Valenzuela-Rodríguez $z^{3, \mathrm{~d}}$

\begin{abstract}
RESUMEN
Con el objetivo de describir las características clínicas y epidemiológicas de la insuficiencia cardiaca aguda (ICA), se realizó un estudio descriptivo en 1075 pacientes de un hospital de Lima. La edad promedio fue 74 años y el $55 \%$ fueron de sexo masculino. El 39\% tuvo ICA con fracción de eyección reducida, el $15 \%$ con fracción de eyección de rango medio y el $46 \%$ con fracción de eyección preservada. Las comorbilidades más frecuentes fueron la hipertensión arterial $(52,6 \%)$ y la enfermedad coronaria (51\%). El $29,2 \%$ de los pacientes tuvo hospitalizaciones previas por ICA. La mediana de la estancia hospitalaria fue de 3 días. La mortalidad intrahospitalaria por todas las causas fue 7,2\%. La hospitalización ocurre, predominantemente, en pacientes de edad avanzada y con múltiples comorbilidades. El bajo uso de fármacos recomendados, conjuntamente con la alta frecuencia de hospitalizaciones previas, probablemente, inciden en la alta tasa de mortalidad registrada en el presente estudio.
\end{abstract}

Palabras clave: Insuficiencia cardiaca, hospitalización, mortalidad intrahospitalaria, epidemiologia, Perú (fuente: DeCS BIREME)

\section{EPIDEMIOLOGICAL CLINICAL CHARACTERISTICS OF ACUTE CARDIAC INSUFFICIENCY IN A TERTIARY HOSPITAL IN LIMA, PERU}

\begin{abstract}
The objective of this study was to describe the clinical and epidemiological characteristics of acute heart failure (AHF) in 1,075 patients from a hospital in Lima, Peru. The average patient age was 74 years and $55 \%$ of study subjects were men. Moreover, $39 \%$ of patients in the sample had AHF with low ejection fraction, $15 \%$ had intermediate ejection fraction, and $46 \%$ had preserved ejection fraction. The most common comorbidities were hypertension $(52.6 \%)$ and coronary disease $(51 \%)$. Moreover, $29.2 \%$ of patients had previous hospitalizations due to AHF. The median hospital stay was 3 days. The rate of inhospital mortality from all causes was $7.2 \%$. Hospitalization was more common in elderly patients with multiple comorbidities. The low use of prescription drugs together with the high rate of previous hospitalizations may explain the high mortality rate reported in this study.
\end{abstract}

Key Words: Heart failure, hospitalization, in-hospital mortality, epidemiology, Peru (source: MeSH NLM)

\section{INTRODUCCIÓN}

Se estima que aproximadamente un millón de hospitalizaciones por insuficiencia cardíaca aguda (ICA) se producen anualmente en los Estados Unidos y se espera que este número se eleve significativamente durante las próximas décadas ${ }^{(1-2)}$. La ICA se ha convertido en la principal causa de hospitalización en personas mayores de 65 años, con altas tasas de mortalidad y reingresos frecuentes ${ }^{(3)}$. Es importante señalar que el costo del tratamiento por ICA está representado, principalmente, por el costo de las hospitalizaciones ${ }^{(4)}$.

Aunque no existe una definición y/o nomenclatura universalmente aceptada para los "síndromes de insuficiencia cardíaca" que requieren hospitalización, probablemente la más aceptada es la preconizada por la Sociedad Europea de Cardiología: «Condición médica que amenaza la vida, que requiere típicamente hospitalización, caracterizada por un inicio rápido o empeoramiento de síntomas y/o signos de insuficiencia cardíaca» ${ }^{(5-7)}$.

En América Latina existe una excesiva carga por ICA que conlleva a altos índices de hospitalización y elevada mortalidad (8). Registros realizados en Chile, Argentina, Brasil y Colombia, demuestran que existen diferencias importantes, aun en países de una misma región, aunque todos coinciden en la alta mortalidad intrahospitalaria y en la baja prescripción de fármacos basados en evidencia ${ }^{(9-12)}$.

\footnotetext{
Hospital Nacional Edgardo Rebagliati Martins. Departamento de Cardiología. Lima, Perú.

Universidad de San Martín de Porres. Facultad de Medicina. Lima, Perú.

Clínica Delgado, Grupo AUNA. Lima, Perú

Médico cardiólogo, máster en Ecocardiografía Transesofágica; ${ }^{\mathrm{b}}$ médico cardiólogo; ${ }^{\mathrm{c}}$ médico residente de Cardiología; ${ }^{\mathrm{d}}$ médico cardiólogo, doctor en Medicina Recibido: 25/05/2017 Aprobado: 25/10/2017 En línea: 15/11/2017
} 
En el año 2012, la ICA fue el principal diagnóstico de egreso en el $16 \%$ de los pacientes hospitalizados en el Servicio de Cardiología del Hospital Nacional Edgardo Rebagliati Martins (HNERM). A pesar del impacto que tiene esta enfermedad en la calidad de vida de las personas y en el pronóstico que impone, aún no se cuenta con información clínico-epidemiológica para ningún establecimiento de salud del tercer nivel en Perú.

El objetivo del presente estudio es describir las características clínicas y epidemiológicas de los pacientes hospitalizados por ICA en un hospital terciario de Lima y, de esta manera, contribuir con iniciativas futuras para mejorar el manejo y los resultados en este grupo de pacientes.

\section{EL ESTUDIO}

Estudio descriptivo que incluyó a pacientes mayores de 18 años hospitalizados por ICA, en el Servicio de Emergencia de Adultos del HNERM, entre diciembre de 2013 y marzo de 2017. Los datos se obtuvieron de las historias clínicas y fueron registrados en una ficha especialmente diseñada para este trabajo. Los pacientes fueron registrados solo una vez durante el curso del estudio. Se incluyen a pacientes hospitalizados con diagnóstico de ICA, según la definición de la Sociedad Europea de Cardiologia ${ }^{(6)}$, independientemente de la etiología o causa de descompensación.

La edad fue analizada como cuantitativa y luego categorizada por grupos etarios. Asimismo, la presión arterial $(\mathrm{mmHg})$, la frecuencia cardíaca (latidos/minuto), la frecuencia respiratoria (respiraciones/minuto), los niveles séricos de sodio $(\mathrm{mEq} / \mathrm{L})$, creatinina $(\mathrm{mg} / \mathrm{dL})$, hemoglobina $(\mathrm{g} / \mathrm{dL})$ y pro-péptido natriurético tipo $B$ (pro-PNB) $(\mathrm{pg} / \mathrm{mL})$ fueron consideradas como variables cuantitativas.

El sexo, las comorbilidades, la presencia de hallazgos en el examen clínico (crepitantes, galope auricular, galope ventricular, ingurgitación yugular o edema), las hospitalizaciones previas, la clase funcional previa según la New York Heart Association (NYHA) (I, II, III y IV), la fracción de eyección del ventrículo izquierdo (FEVI) (fracción de eyección reducida $<40 \%$, en rango medio $40-49 \%$ o preservada $\geq 50 \%$ ) ${ }^{(7)}$, la medicación recibida y la mortalidad intrahospitalaria, fueron analizados de manera cualitativa.

El estudio fue revisado y aprobado por los comités de investigación y ética del HNERM; al usarse solo los registros de las historias clínicas no fue necesario la firma de un consentimiento informado; solo los investigadores tuvieron acceso a la información para asegurar la confidencialidad de los pacientes. La información se almacenó en una base de datos elaborada en Excel 2010 $\mathrm{y}$, posteriormente, se procesó con el programa estadístico SPSS versión 22. Las variables continuas se expresaron como media y desviación estándar o mediana y rango intercuartílico; para evaluar la distribución de las variables

\section{MENSAJES CLAVE}

Motivación para realizar el estudio. Describir las características clínico epidemiológicas de la insuficiencia cardiaca aguda en pacientes hospitalizados en un hospital terciario de Lima, Perú.

Principales hallazgos. La hospitalización por insuficiencia cardiaca se produce, principalmente, en personas de edad avanzada, con múltiples comorbilidades. La alta frecuencia de hospitalizaciones previas, la estancia prolongada y el bajo uso de fármacos recomendados, probablemente, se asocien a la alta tasa de mortalidad registrada.

Implicancias. El impacto en salud pública, radica en el alto costo que representa la hospitalización de pacientes con ICA, debido a su edad, comorbilidad múltiple e inadecuado manejo clínico.

se utilizó la prueba de Kolmogorov-Smirnov con corrección de Lilliefors. Las variables categóricas se expresaron como valores absolutos y frecuencias relativas. Las diferencias en la administración de medicamentos al ingreso y al alta hospitalaria fueron evaluadas con el test de McNemar, considerándose un valor de $p<0,05$ como estadísticamente significativo.

\section{HALLAZGOS}

Se registraron 1075 pacientes que cumplieron el criterio de inclusión; la edad promedio fue 73,6 \pm 10,9 años, siendo $85,7 \%$ mayor de 60 años y $31,2 \%$ mayor de 80 años; el $55 \%$ de los pacientes fue de sexo masculino.

Las comorbilidades más frecuentes fueron la hipertensión arterial $(52,6 \%)$ y la enfermedad coronaria (51\%). La anemia, la enfermedad renal crónica y la diabetes representaron cada una, aproximadamente una tercera parte de la población estudiada. La clase funcional previa NYHA predominante fue la clase II (45,6\%), seguida por la clase funcional III $(42,7 \%)$ (Tabla 1$)$.

La población estudiada presentó una mediana de presión arterial sistólica de 129,5 $\mathrm{mmHg}$, con taquicardia y taquipnea predominantes. La presencia de crepitantes se detectó en más de dos tercios de los pacientes $(70,1 \%)$ y la mitad de ellos tuvo ingurgitación yugular y/o edema de miembros inferiores (51,1 y 49,7\%, respectivamente). El hallazgo de tercer o cuarto ruido cardiaco fue menos frecuente.

Del total de pacientes registrados, solo 535 (49,7\%) tuvieron evaluación por ecocardiografía de la fracción de eyección del ventrículo izquierdo (FEVI); la distribución de la FEVI en este grupo, según la clasificación vigente de la Sociedad Europea de Cardiología ${ }^{(7)}$, se muestra en la tabla 2.

La medicación previa para ICA usada con mayor frecuencia fueron los inhibidores de enzima convertidora deangiotensina (IECA)/bloqueadores de los receptores de angiotensina 
Tabla 1. Características basales y de estancia hospitalaria en pacientes con insuficiencia cardiaca aguda. Hospital Nacional Edgardo Rebagliati Martins, 2013 a 2017

\begin{tabular}{|c|c|}
\hline Variable & $\mathrm{n}=1075$ \\
\hline Media de la edad (DE) & $73,6 \pm 10,9$ \\
\hline \multicolumn{2}{|l|}{ Grupo etario (\%) (años) } \\
\hline$<40$ & $2(0,2)$ \\
\hline $40-60$ & $152(14,1)$ \\
\hline $61-80$ & $586(54,5)$ \\
\hline$>80$ & $335(31,2)$ \\
\hline \multicolumn{2}{|l|}{ Sexo $(\%)$} \\
\hline Masculino & $591(55,0)$ \\
\hline Femenino & $484(45,0)$ \\
\hline \multicolumn{2}{|l|}{ Comorbilidades $(\%)^{*}$} \\
\hline Hipertensión arterial & $565(52,6)$ \\
\hline Enfermedad coronaria & $548(51,0)$ \\
\hline Arritmia cardiaca & $408(38,0)$ \\
\hline Enfermedad renal crónica & $322(30,0)$ \\
\hline Diabetes mellitus & $314(29,2)$ \\
\hline Anemia & $312(29,0)$ \\
\hline Dislipidemia & $168(15,6)$ \\
\hline Valvulopatías & $130(12,1)$ \\
\hline \multicolumn{2}{|l|}{ Clase funcional previa (NYHA) (\%) } \\
\hline Clase I & $55(5,1)$ \\
\hline Clase II & $490(45,6)$ \\
\hline Clase III & $459(42,7)$ \\
\hline Clase IV & $71(6,6)$ \\
\hline Insuficiencia cardiaca previa (\%) & $662(61,6)$ \\
\hline Sin hospitalización previa por ICA (\%) & $761(70,8)$ \\
\hline Con hospitalización previa por ICA (\%) & $314(29,2)$ \\
\hline 1 hospitalización & $91(8,4)$ \\
\hline 2 hospitalizaciones & $79(7,4)$ \\
\hline 3 hospitalizaciones & $87(8,1)$ \\
\hline 4 hospitalizaciones & $52(4,8)$ \\
\hline 5 o más hospitalizaciones & $5(0,5)$ \\
\hline Mediana de la estancia hospitalaria en días (RIC) & $3(2-7)$ \\
\hline \multicolumn{2}{|l|}{ Estancia hospitalaria (\%) (días) } \\
\hline$\leq 3$ & $657(61,1)$ \\
\hline $4-7$ & $164(15,3)$ \\
\hline $8-14$ & $147(13,7)$ \\
\hline $15-28$ & $94(8,7)$ \\
\hline$>28$ & $13(1,2)$ \\
\hline
\end{tabular}

DE: desviación estándar, NYHA: New York Heart Association, RIC:rango intercuartílico, ICA: insuficiencia cardiaca aguda

* Se puede presentar más de un antecedente de enfermedad

(BRA) (51,6\%), diurético de asa como furosemida $(41,2 \%)$, beta bloqueadores $(31,7 \%)$, espironolactona $(14,9 \%)$ y digoxina $(13,9 \%)$. Al alta hospitalaria, el uso de IECA/BRA y betabloqueadores aumentó a 75,5 y 64,1\%, respectivamente, el uso de espironolactona también aumentó en menor cuantía y se produjo un descenso en el uso de furosemida y digoxina (Tabla 3 ).
Tabla 2. Hallazgos del examen clínico de ingreso y exámenes auxiliares en pacientes con insuficiencia cardiaca aguda. Hospital Nacional Edgardo Rebagliati Martins, 2013 a 2017

\begin{tabular}{|c|c|}
\hline Variable & $\mathrm{n}=1075$ \\
\hline Mediana de la PAS en mmHg (RIC) & $126(109-139)$ \\
\hline Mediana de la FC en lat/min (RIC) & $92(80-110)$ \\
\hline Mediana de la FR en resp/min (RIC) & $22(20-26)$ \\
\hline \multicolumn{2}{|l|}{ Examen clínico (\%) } \\
\hline Crepitantes & $754(70,1)$ \\
\hline Galope ventricular & $103(9,6)$ \\
\hline Galope auricular & $61(5,7)$ \\
\hline Ingurgitación yugular & $549(51,1)$ \\
\hline Edema & $534(49,7)$ \\
\hline Mediana del sodio en mEq/l (RIC) & $139(136-141)$ \\
\hline Mediana de la creatinina en mg/dL (RIC) & $1(0,8-1,6)$ \\
\hline Mediana de la hemoglobina en g/dL (RIC) & $12,5(10,8-13,1)$ \\
\hline Mediana de pro-PNB en pg/mL (RIC) & $3310(977-19347)$ \\
\hline FEVI $(\%)^{*}$ & $n=535$ \\
\hline$<40$ (ICFEr) & $209(39,1)$ \\
\hline $40-49$ (ICFEm) & $80(14,9)$ \\
\hline$\geq 50$ (ICFEp) & $246(46,0)$ \\
\hline
\end{tabular}

PAS: presión arterial sistólica. FC: frecuencia cardiaca. FR: frecuencia respiratoria. RIC: rango intercuartílico. FEVI: fracción de eyección del ventrículo izquierdo, pro-PNB: pro-péptido natriurético tipo $B$. ICFEr: insuficiencia cardiaca con fracción de eyección reducida. ICFEm: insuficiencia cardiaca con fracción de rango medio. ICFEp: insuficiencia cardiaca con fracción de eyección preservada.

${ }^{*} \mathrm{FEVI}$ solo se realizó en 535 pacientes

El 29,2\% de los pacientes tuvieron hospitalizaciones previas por ICA y el $13,4 \%$ tuvo tres o más hospitalizaciones previas. La estancia hospitalaria tuvo una mediana de tres días, el $23,6 \%$ de los pacientes tuvo una estancia hospitalaria mayor a siete días. El porcentaje de complicaciones relacionadas a ICA fue de $15 \%$ y la mortalidad intrahospitalaria por todas las causas fue de $7,2 \%$.

\section{DISCUSIÓN}

Las características del síndrome de ICA pueden tener diferencias regionales significativas ${ }^{(9-14)}$, por lo que es importante tomar conocimiento de la realidad local. Nuestro trabajo representa, hasta donde tenemos conocimiento, el primer registro de ICA en Perú y proporciona información inédita sobre esta importante patología cardiovascular.

El presente registro muestra que la hospitalización por ICA ocurre preferentemente en una población de edad avanzada, siendo las principales causas, la hipertensión arterial y la enfermedad coronaria. Estas frecuencias son similares a lo reportado en registros de Estados Unidos y Europa (15-17). Sin embargo, son diferentes a lo reportado en los registros latinoamericanos (9-14). Actualmente la enfermedad valvular continúa siendo una causa importante de hospitalización en nuestra 
Tabla 3. Diferencias en la administración de medicamentos para insuficiencia cardiaca al ingreso y al alta hospitalaria. Hospital Nacional Edgardo Rebagliati Martins, 2013 a 2017

\begin{tabular}{lccc}
\hline \multirow{2}{*}{ Medicamentos } & Al ingreso & Al alta (\%) & \multirow{2}{*}{ Valor de $\mathbf{p}^{*}$} \\
\cline { 2 - 3 } & $\mathbf{n}(\%)$ & $\mathbf{n}(\%)$ & $<0,001$ \\
IECA/BRA & $555(51,6)$ & $612(75,5)$ & $<0,001$ \\
Espirobloqueador & $341(31,7)$ & $689(64,1)$ & 0,125 \\
Furosemida & $160(14,9)$ & $208(19,3)$ & 0,031 \\
Digoxina & $443(41,2)$ & $377(35,1)$ & 0,5 \\
\hline
\end{tabular}

IECA: inhibidores de enzima convertidora de angiotensina, BRA: bloqueadores de los receptores de angiotensina

*Test de McNemar

población, probablemente debido a la alta frecuencia de fiebre reumática en la región ${ }^{(8)}$. Comorbilidades como la diabetes mellitus, la enfermedad renal crónica y la anemia estuvieron presentes en un rango similar al encontrado en la literatura ${ }^{(18,19)}$.

La frecuencia de FEVI de nuestro registro es bastante menor a la reportada en países desarrollados ${ }^{(15-17)}$, pero es muy similar a lo descrito en el contexto chileno ${ }^{(9)}$, lo que podría deberse a la escasez de recursos y equipamiento en la realidad latinoamericana. Encontramos que la ICA con fracción de eyección preservada (ICFEp) representa cerca del $50 \%$ de los casos, y la ICA con fracción de eyección reducida (ICFEr) representa aproximadamente un $40 \%$, similar a lo descrito en la literatura ${ }^{(18)}$.

Las guías actuales de manejo de ICA recomiendan que los pacientes con ICFEr deben recibir terapia combinada con IECA/BRA, betabloqueadores y un antagonista de aldosterona $^{(5,7)}$. Sin embargo, pese a la robusta evidencia que demuestra una reducción de mortalidad con esta terapia, su uso en la práctica diaria dista de tener la frecuencia esperada, aun en los países desarrollados ${ }^{(9-13,20)}$. Esta realidad se confirma en el presente trabajo, pues se encontraron bajos porcentajes de indicación de las terapias recomendadas por las guías, que se mantienen, incluso, al alta hospitalaria, especialmente en el uso de beta bloqueadores $(64,1 \%)$ y antagonistas de aldosterona (19,3\%), pese al significativo incremento de su uso, producido durante la hospitalización.

La mortalidad intrahospitalaria encontrada en nuestra serie es bastante mayor que la reportada en los registros norteamericanos ${ }^{(15,16)}$ y chilenos (9), aunque considerablemente menor a lo reportado por un registro brasileño y uno internacional de reciente publicación ${ }^{(11,13)}$; lo que demuestra la gran variabilidad existente, inclusive entre países de una misma región.

Existen diferentes aspectos que podrían explicar la alta tasa de mortalidad en el presente trabajo. Primeramente, la hospitalización previa y la duración del internamiento, que imponen un pronóstico sombrío a los pacientes con ICA $(3,18)$; en nuestro registro, casi la tercera parte de los pacientes tenían hospitalizaciones previas, con casi un $18 \%$ con tres o más hospitalizaciones, y un $23 \%$ con más de siete días de estancia hospitalaria. Asimismo, la baja prescripción de fármacos indicados para el tratamiento de ICFEr y la pobre adherencia al tratamiento, común en personas de edad avanzada, contribuyen al empeoramiento de la enfermedad y la necesidad de hospitalización. Finalmente, existe en la realidad regional una menor disponibilidad de recursos que podrían incidir negativamente en la evolución de los pacientes hospitalizados por ICA.

El presente estudio tiene como limitaciones, el haber sido realizado en un centro único, por lo que representa solo una parte de la realidad de la ICA en el Perú, por ello, sus resultados no pueden ser generalizados. Adicionalmente, no se contaron con registros electrocardiográficos, ni ecocardiográficos, aparte de la FEVI, que pudieron haber sido de gran aporte para el estudio.

Se concluye, que la hospitalización por ICA ocurre, predominantemente, en pacientes de edad avanzada, con múltiples comorbilidades. La ICFEp representa aproximadamente la mitad de los internamientos por ICA. El bajo uso de fármacos recomendados en las guías actuales de manejo de ICA, conjuntamente con la alta frecuencia de hospitalizaciones previas, probablemente inciden en la alta tasa de mortalidad registrada en el presente estudio.

Contribuciones de autoría: MP ha participado en la concepción, análisis e interpretación de datos y redacción del artículo, PASS ha participado en la recolección, análisis e interpretación de los datos, MPR ha participado en la recolección de datos, JSRV, MJC y GVR han participado en la concepción del artículo. Todos han aprobado la versión final del artículo.

Fuentes de financiamiento: Autofinanciado

Declaración de conflictos de interés: Ninguno 


\section{REFERENCIAS BIBLIOGRÁFICAS}

1. Go AS, Mozaffarian D, Roger VL, Benjamin EJ, Berry JD, Blaha MJ, et al. American Heart Association Statistics Committee and Stroke Statistics Subcommittee. Heart disease and stroke statistics-2014 update: a report from the American Heart Association. Circulation 2014;129(3):e28-e292. doi: 10.1161/01. cir.0000441139.02102.80

2. Heidenreich PA, Albert NM, Allen LA, Bluemke DA, Butler J, Fonarow GC, et al. American Heart Association Advocacy Coordinating Committee; Council on Arteriosclerosis, Thrombosis and Vascular Biology; Council on Cardiovascular Radiology and Intervention; Council on Clinical Cardiology; Council on Epidemiology and Prevention; Stroke Council. Forecasting the impact of heart failure in the United States: a policy statement from the American Heart Association. Circ Heart Fail 2013;6(3):606-19. doi: 10.1161/HHF.0b013e318291329a.

3. Carson PE, Anand IS, Win S, Rector T, Haass M, Lopez-Sendon J, et al. The hospitalization burden and posthospitalization mortality risk in heart failure with preserved ejection fraction: results from the I-PRESERVE trial (Irbesartan in Heart Failure and Preserved Ejection Fraction). JACC Heart Fail. 2015;3:429-441. doi: 10.1016/j. jchf.2014.12.017.

4. O'Connell JB. The economic burden of heart failure. Clin Cardiol. 2000;23(Suppl 3):III6-10

5. Yancy CW, Jessup M, Bozkurt B, Butler J, Casey DE, Drazner MH, et al. 2013 ACCF/AHA guideline for the management of heart failure: a report of the American College of Cardiology Foundation/American Heart Association Task Force on Practice Guidelines. J Am Coll Cardiol 2013;62:147-239. doi: 10.1016/j. jacc.2013.05.019.

6. McMurray JJV, Adamopoulus S, Anker SD, Auricchio A, Bohm M, Dickstein $\mathrm{K}$, et al. ESC Guidelines for the diagnosis and treatment of acute and chronic heart failure 2012: The Task Force for the Diagnosis and Treatment of Acute and Chronic Heart Failure 2012 of the European Society of Cardiology. Developed in collaboration with the Heart Failure Association (HFA) of the ESC. Eu Heart J. 2012;33(14):1787-847. doi: 10.1093/eurheartj/ehs104.
7. Ponikowski P, Voors AA, Anker SD, Bueno H, Cleland JG, Coats AJ, et al. 2016 ESC Guidelines for the diagnosis and treatment of acute and chronic heart failure: The Task Force for the diagnosis and treatment of acute and chronic heart failure of the European Society of Cardiology (ESC)Developed with the special contribution of the Heart Failure Association (HFA) of the ESC. Eur Heart J. 2016;37(27):2129-200. doi: 10.1093/ eurheartj/ehw128.

8. Bocchi EA, Arias A, Verdejo H, Diez M, Gómez E, Castro P, et al. The reality of heart failure in Latin America. J Am Coll Cardiol. 2013;62(11):949-58. doi: 10.1016/j.jacc.2013.06.013.

9. Castro P, Vukasovic JL, Garcés E, Sepúlveda L, Ferrada M, Alvarado S, et al. Insuficiencia cardíaca en hospitales chilenos: resultados del Registro Nacional de Insuficiencia Cardíaca, Grupo ICARO. Rev Med Chil 2004;132(6):655-62

10. Perna ER, Barbagelata A, Grinfeld L, Ben MG, Canella JPC, Bayol PA, et al. Overview of acute decompensated heart failure in Argentina: Lessons learned from 5 registries during the last decade. Am Heart J. 2006; 151(1):84-91.

11. Albuquerque DC, Neto JD, Bacal F, Rohde LE, Bernardez-Pereira S, Berwanger $\mathrm{O}$, et al. I Brazilian registry of heart failure-clinical aspects, care quality and hospitalization outcomes. Arq Bras Cardiol 2015;104(6):433-42. doi: 10.5935/abc.20150031.

12. Senior JM, Saldarriaga C, Rendon JA. Descripción clínico-epidemiológica de los pacientes con falla cardiaca aguda que consultan al servicio de urgencias. Acta Med Colomb. 2011;36(3):125-129.

13. Dokainish H, Teo K, Zhu J, Roy A, AlHabib KF, ElSayed A, et al. Global mortality variations in patients with heart failure: results from the International Congestive Heart Failure (INTER-CHF) prospective cohort study. The Lancet Global Health. 2017;5(7):e665-e672. doi: 10.1016/S2214-109X(17)30196-1.

14. Callender T, Woodward M, Roth G, Farzadfar F, Lemarie JC, Gicquel S, et al. Heart Failure Care in Low- and Middle-Income Countries: A Systematic Review and Meta-Analysis. PLoS Med. 2014;11(8):e1001699. doi: 10.1371/ journal.pmed.1001699.
15. Adams KF, Fonarow GC, Emerman CL, LeJemtel TH, Costanzo MR, Abraham WT, et al. Characteristics and outcomes of patients hospitalized for heart failure in the United States: rationale, design, and preliminary observations from the first 100,000 cases in the Acute Decompensated Heart Failure National Registry (ADHERE). Am Heart J. 2005; 149(2):209-16.

16. Fonarow GC, Stough WG, Abraham WT, Albert NM, Gheorghiade M, Greenberg $\mathrm{BH}$, et al. Characteristics, treatments, and outcomes of patients with preserved systolic function hospitalized for heart failure: a report from the OPTIMIZE-HF Registry. J Am Coll Cardiol. 2007;50(8):768-77. doi: 10.1016/j.jacc.2007.04.064.

17. Nieminen MS, Brutsaert D, Dickstein K, Drexler H, Follath F, Harjola VP, et al. EuroHeart Failure Survey II (EHFS II): a survey on hospitalized acute heart failure patients: description of population. Eur Heart J 2006;27:2725-2736.

18. Kapoor JR, Kapoor R, Ju C, Heidenreich PA, Eapen ZJ, Hernandez AF, et al. Precipitating clinical factors, heart failure characterization, and outcomes in patients hospitalized with heart failure with reduced, borderline, and preserved ejection fraction. JACC Heart Fail. 2016;4(6):464-72. doi: 10.1016/j. jchf.2016.02.017.

19. Groenveld HF, Januzzi JL, Damman $\mathrm{K}$, van Wijngaarden J, Hillege $\mathrm{HL}$, van Veldhuisen D J, et al. Anemia and mortality in heart failure patients: a systematic review and meta-analysis. J Am Coll Cardiol. 2008 ;52(10):818-27. doi: 10.1016/j.jacc.2008.04.061.

20. Maggioni AP, Anker SD, Dahlström U, Filippatos G, Ponikowski P, Zannad $\mathrm{F}$, et al. Are hospitalized or ambulatory patients with heart failure treated in accordance with European Society of Cardiology guidelines? Evidence from 12440 patients of the ESC Heart Failure Long-Term Registry. Eur J Heart Fail. 2013;15(10):1173-84. doi: 10.1093/ eurjhf/hft134

\section{Correspondencia: Marcos Pariona}

Dirección: Hospital Edgardo Rebagliati Martins. Departamento de Cardiología. Av. Edgardo Rebagliati 490. Jesús María. Lima,Perú Teléfono: (+51) 949209438

Correo electrónico: marcospariona@gmail.com 